\title{
A contribuição da genealogia acadêmica para a construção de indicadores bibliométricos
}

\author{
Vanessa Paula Alves de Moura \\ Mestre; Universidade Federal de São Carlos, São Carlos, SP, Brasil; \\ Vanessa-adm@hotmail.com \\ Leandro Innocentini Lopes de Faria \\ Doutor; Universidade Federal de São Carlos, São Carlos, SP, Brasil; \\ leandro@ufscar.br
}

\begin{abstract}
Resumo: A medição da produção científica por meio da bibliometria, que permite avaliar o desempenho dos pesquisadores e os impactos de suas produções, é objeto de estudo muito explorado na Ciência da Informação. A genealogia acadêmica, por outro lado, permite estudar a herança intelectual que se resulta dos relacionamentos entre orientadores e seus alunos. Estudos recentes no Brasil sobre a genealogia acadêmica utilizam como base de dados a Plataforma Lattes, extraindo informações que podem ser utilizadas para diversas análises. Diante disso, este trabalho tem por objetivo investigar se a aplicação da genealogia acadêmica e da análise bibliométrica em conjunto pode trazer informações complementares sobre a atuação de um programa de pós-graduação na formação de pesquisadores e produção científica, realizando um estudo de caso baseado no Programa de Pós-Graduação em Engenharia de Materiais da Universidade Federal de São Carlos. A metodologia utilizada baseia-se no método de identificação de grafos de genealogia acadêmica em conjunto com as ferramentas SyncLattes, VantagePoint e Microsoft Excel. Como resultados são apresentados indicadores bibliométricos sobre o programa e seus descendentes, que podem contribuir na elaboração de estratégias para a autoavaliação e o desempenho dos programas de pós-graduação.
\end{abstract}

Palavras-chave: Bibliometria. Genealogia acadêmica. Produção científica. Indicadores de ciência e tecnologia. Engenharias II.

\section{Introdução}

O homem, desde a sua origem, busca conhecer os objetos que o cerca, a princípio motivado pela necessidade de controle dos fenômenos naturais, para garantir a sua própria sobrevivência biológica e, posteriormente, pela busca do conhecimento. A compreensão do mundo de forma mais ampla, somada à necessidade de troca de informações, tem como resultado a elaboração de sistemas mais estruturados de organização do conhecimento (ARAÚJO, 2006). 
O surgimento da ciência moderna vai além do desenvolvimento de procedimentos de investigação e descoberta de novos fenômenos, preocupa-se, também, com o desenvolvimento de teorias capazes de explicá-los (CHIBENI, 2019).

Desde o seu surgimento, cada vez mais, a ciência desempenha um papel extremamente importante para a sociedade, criando conhecimento e melhorando a qualidade de vida das pessoas à medida que possibilita avanços nos campos da saúde, da alimentação, do ambiente, da tecnologia, entre outros (UNESCO, 2019).

Há uma relação direta entre a ciência e as atividades de pesquisas. A pesquisa é um processo que auxilia a ciência a entender os fatos que acontecem diante das relações sociais, que estão em constantes mudanças (SOUZA, 2011).

Um grupo social formado por indivíduos que tem como profissão a pesquisa científica forma uma comunidade científica. Dentro dessas comunidades, o principal sistema é o de doação no qual, geralmente, o pesquisador transfere para a sua comunidade as informações de sua pesquisa, sem qualquer interesse monetário. Em contrapartida, a comunidade científica, oferece a esse pesquisador a confirmação de que ele é um cientista, tornando-o reconhecido conforme os trabalhos desenvolvidos e publicados (LE COADIC, 1996).

Os pesquisadores podem tornar acessíveis os resultados do seu trabalho de investigação nas mais diversas áreas do conhecimento, por meio de suas publicações científicas, que consistem na divulgação dos resultados, que podem ser comunicados sob diferentes formas de publicações (BIBLIOTECA DA FEUP, 2019).

Atualmente, os artigos científicos ganham destaque, tendo significativa relevância para a ciência. São estudos publicados em periódicos científicos nas diferentes disciplinas e áreas temáticas, constituindo-se como o principal meio de comunicação dos resultados da pesquisa científica. Os primeiros periódicos científicos surgiram há mais de 350 anos e são um dos principais meios de comunicação dos resultados de pesquisa científica (SPINAK; PACKER, 2015). 
Depois que uma pesquisa é publicada, outro pesquisador pode acessar essas informações e utilizá-las como subsídios para construir um novo conhecimento e assim sucessivamente. É essa retroalimentação contínua, que faz a ciência evoluir (ROSA e BARROS, 2018).

Durante todo o decorrer da história, nas mais diversas áreas, o homem tem buscado formas para classificar, registrar, organizar e difundir a informação. Para tanto, foi criada uma área específica para tratar de problemas relativos à informação, enquanto um fenômeno social. Desta forma, surge a Ciência da Informação, com a perspectiva de atender à necessidade de se organizar o conhecimento e a disseminação da informação (SILVA e FREIRE, 2012).

Já no início do século XX, cientistas começaram a perceber que a quantidade de conhecimento científico gerado, continuamente, ultrapassa a sua capacidade de leitura. O volume do conhecimento gerado por meio de novos periódicos e publicações, diante da impossibilidade de adquirir todos, levou os profissionais da informação a buscarem maneiras de selecionar os mais significativos, como a cientometria, infometria e bibliometria (FARIA, 2001).

De acordo com a Plataforma Sucupira (2020) a Ciência da Informação é uma área da Ciências Sociais Aplicadas e possui três subáreas: Biblioteconomia, Arquivologia e Teoria da Informação. A Bibliometria é uma especialidade da Biblioteconomia e de segundo Faria (2001), é uma técnica de contagem de toda e qualquer informação escrita.

A bibliometria pode ser definida como a análise quantitativa de publicações, autores e referências bibliográficas, um campo que, gradativamente, tornou-se significativo na atividade de experimentação e pesquisa. Com o passar dos anos, a bibliometria extrapolou os limites das bibliotecas e passou, também, a considerar revistas acadêmicas e artigos de periódicos, autores e instituições, referências bibliográficas, citações, agradecimentos e patentes, sendo beneficiada com o surgimento da internet (SUGIMOTO, 2014).

Segundo o mesmo autor, o interesse pela bibliometria por órgãos de financiamento, corporações e universidades nunca foi tão grande, o que não é por acaso, já que um dos critérios utilizados por governos e órgãos 
multinacionais, na decisão para direcionar seus recursos destinados à pesquisa e desenvolvimento, é a avaliação do trabalho científico, por meio de indicadores quantitativos.

A produção científica tornou-se um instrumento utilizado no momento de decidir para onde serão destinados os recursos à pesquisa, que são limitados e competem com os demais setores de investimento público (VELHO, 2011).

Apesar da importância das métricas de produção, segundo Ribeiro(2007), o principal "produto" de um programa de pós-graduação não são os artigos, os livros, as teses e as dissertações, mas sim os mestres e doutores formados pelo programa dos quais, espera-se, a continuidade na pesquisa. $\mathrm{O}$ impacto social é um dos critérios avaliados nos cursos de mestrado e doutorado pela Coordenação de Aperfeiçoamento de Pessoal de Nível Superior (CAPES),Fundação do Ministério da Educação (MEC), responsável pela consolidação do regulamento da pós-graduação no Brasil.

A problemática está em fazer o mapeamento de todos os egressos de um programa, de forma que muitos deles, não mantêm o vínculo com a instituição após a sua formação e muitos de seus dados, como atuação atual, são voláteis (LIMA, 2015).

Diante deste cenário, esta pesquisa buscou meios de como fazer isso por meio da genealogia acadêmica, que, segundo Damaceno et. al. (2019),busca compreender a ciência por meio da relação orientador e orientado. As árvores genealógicas permitem obter uma melhor compreensão da configuração e evolução dos pesquisadores e dos respectivos grupos, auxiliando agências de financiamento e instituições acadêmicas para avaliar a formação dos recursos humanos, além de complementar a avaliação dos resultados da pesquisa.

Para tanto, estudos recentes utilizam em suas pesquisas a Plataforma Lattes como base de dados na construção da genealogia acadêmica, que podem ser conferidos em Oliveira et. al. (2018),Moreira et. al. (2018) e Dores et al.(2017).

O Currículo Lattes é adotado pela maioria das instituições de fomento, universidades e institutos de pesquisas do país. Apresenta ricas, confiáveis e abrangentes informações referentes à vida pregressa e atual dos estudantes e 
pesquisadores do país. Por esse motivo, atualmente é um elemento obrigatório para a análise de mérito e competência das ações de financiamentos na área da ciência e tecnologia (CNPq, 2019).

No entanto, como os dados dos Currículos Lattes são indexados manualmente pelos pesquisadores, de forma não padronizada, existem circunstâncias que dificultam a tarefa de extração, como por exemplo, a inexistência de alguns currículos, divergência ou abreviações nos nomes das instituições, dos cursos, dos orientadores e orientados e currículos com dados incompletos ou desatualizados, dificultando a associação do ID Lattesdos pesquisadorese a construção das relações (ROSSI, DAMACENO e MENACHALCO, 2018).

A utilização da genealogia para documentar indivíduos e seus relacionamentos tem como objetivo a obtenção de conhecimento sobre a origem, evolução e disseminação de grupos inter-relacionados, auxiliando o entendimento da formação e as tendências futuras de grupos de pessoas (ROSSI e MENA-CHALCO, 2014).

É possível identificar várias formas de herança por meio do estudo das genealogias e, para isso, utilizam-se o heredograma, também conhecido como árvore genealógica, que é a representação gráfica dos indivíduos relacionados por parentesco (INSTITUTO DE CIÊNCIAS BIOLÓGICAS, 2019).

O estudo das árvores acadêmicas permite conhecer a cronologia das ideias de uma área de pesquisa, associando-as aos principais autores na formação dessas ideias e seus descendentes científicos (ANDRAOS, 2005).

Contudo, o que garante a continuidade da linhagem acadêmica é a produção de novos doutores, porém é necessário que estes permaneçam na academia após a obtenção do título. O pressuposto implícito, nas concepções de reprodução disciplinar, é de que os alunos se tornarão mestres na mesma disciplina em que foram treinados(SUGIMOTO et al., 2011).

Desta forma, a genealogia fornece um meio de medir e analisar a propagação do conhecimento científico, a obtenção de insumos quantitativos para mensurar as interações acadêmicas e a migração de área de um acadêmico. A migração de área por um acadêmico tem um impacto direto no cenário do 
conhecimento, mudando a trajetória esperada da próxima geração de mestres (SUGIMOTO, 2014).

Para o trabalho de um cientista viver além de seu tempo é preciso que ele preencha a próxima geração de acadêmicos com pessoas que ele orientou, para que suas ideias, contribuições e visões continuem influenciando o pensamento científico. A vida de um erudito é finita, mas sua contribuição é ampliada, reforçada e prolongada por meio de sucessivas gerações de aprendizes (ANDRAOS, 2005).

Segundo Rossi e Mena-Chalco (2014), a análise genealógica acadêmica se mostra importante por apresentar a possibilidade de avaliar o impacto das orientações acadêmicas no desenvolvimento científico de áreas específicas do conhecimento e na identificação dos principais atores, ou grupo de maior relevância na proliferação do conhecimento.

Diante do potencial da aplicação da genealogia acadêmica e da importância das métricas para a atuação dos programas de pós-graduação, este trabalho tem por objetivo investigar se a aplicação da genealogia acadêmica e da análise bibliométrica em conjunto, pode trazer informações complementares sobre a contribuição de um programa de pós-graduação na formação de pesquisadores e produção científica, realizando um estudo de caso baseado no Programa de Pós-Graduação em Engenharia de Materiais da Universidade Federal de São Carlos (PPGCEM/UFSCar) durante toda a sua existência.

Para atingir este objetivo foi utilizado o método de identificação de grafos de genealogia acadêmica,desenvolvido no trabalho de Damaceno, Rossi e Mena-Chalco (2017), que tem como base de dados a Plataforma Lattes.Adicionalmente,foi utilizada a ferramenta computacional SyncLattes para a extração das publicações científicas. Para a análise dos dados e construção de indicadores bibliométricos foram usadas as ferramentas VantagePoint e Microsoft Excel.

\section{Método e desenvolvimento da pesquisa}

A metodologia para esta pesquisa é baseada em uma abordagem quantitativa. Quanto à natureza, trata-se de uma pesquisa aplicada, uma vez que vai gerar 
conhecimentos para aplicações práticas na solução de problemas específicos. Quanto aos objetivos é uma pesquisa exploratória.

Para estudo de caso foi escolhido o PPGCEM/UFSCar,devido ao programa ter mais de 40 anos, sendo o pioneiro no Brasil, contribuindo, de maneira significativa,na disseminação da Engenharia de Materiais no país com expressiva atuação na formação de mestres (SAVIGNANO, 2012).

A extração de dados, realizada em janeiro de 2020, e construção da genealogia acadêmica do programa, a partir da Plataforma Lattes, foi baseada no método de identificação de grafos de genealogia acadêmica desenvolvido por Damaceno, Rossi e Mena-Chalco (2017).

O problema deste método é que ele cria a genealogia acadêmica dos pesquisadores considerando todas as relações e, como resultado, traz uma enorme quantidade de dados dos orientadores, orientados e de suas relações. Esses dados, são brutos, ou seja, necessitam de procedimentos e análises para serem interpretados (DAMACENO, ROSSI e MENA-CHALCO, 2017).

Desta forma, mapear todos os acadêmicos que têm sua origem no PPGCEM/UFSCar, sem ruídos de outras relações que não pertencem ao programa, foi um desafio encontrado durante este estudo.No entanto, de acordo com Rossi; Damaceno e Mena-Chalco (2018), a estruturação e análise desses dados representam atuais oportunidades de pesquisa e aplicação que envolvem as áreas de Ciência da Computação, Ciência da Informação e Política Científica.

Diante disso, buscou-se minimizar esses esforços, adotando como procedimento a execução do método por duas vezes: 1) a partir dos docentes que atuaram e atuam no programa e 2) a partir dos seus orientados. Em ambas, foram desconsideradas as ascendências, e todo processo de extração de dados foi feito de forma automática.

Portanto, o método de pesquisa,foi dividido em cinco etapas esquematizadas na Figura 1. 


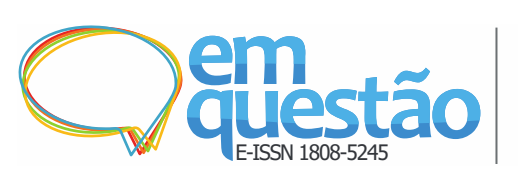

Figura 1- Fluxograma do procedimento da pesquisa

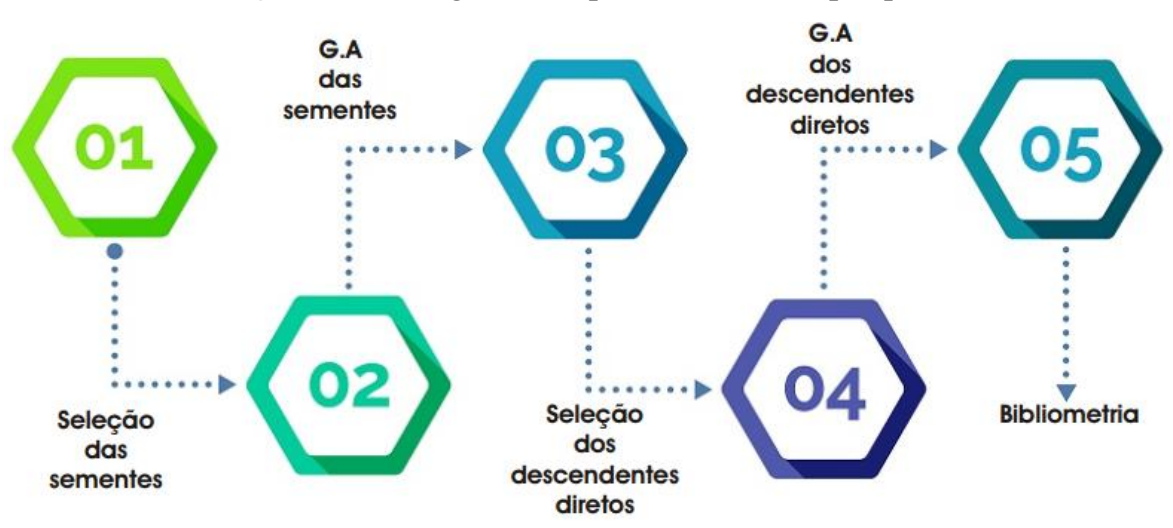

Fonte: Elaborado pelos autores.

A primeira etapa refere-se à seleção das sementes junto à UFSCar. O levantamento de todos os docentes que atuaram e ainda atuam no programa e seus respectivos identificadores únicos na Plataforma Lattes (ID Lattes), foram adquiridos junto à Secretaria Geral de Planejamento e Desenvolvimento Institucionais (SPDI) da instituição, totalizando cinquenta e oito docentes entre os anos de 1979 a 2019. Esses pesquisadores são a origem do conhecimento do programa, os quais a partir de agora, serão denominados como "sementes".

A segunda etapa consiste em gerar a genealogia acadêmica pela primeira vez, a partir dos ID Lattes das sementes do programa. Alguns dos resultados da genealogia acadêmica gerada são dispostos em uma planilha denominada 7_arestas.

A terceira etapa consiste em selecionar apenas os descendentes diretos do programa, ou seja, aqueles que possuem a titulação de mestre ou doutor no PPGCEM/UFSCar. Para tanto, foi feita uma classificação, utilizando os filtros do Microsoft Excel,aplicados na planilha 7_arestas, de acordo com os seguintes critérios:

1) Seleção da instituição: na filtragem deste campo foram considerados os seguintes termos atribuídos no preenchimento do Currículo Lattes: "Universidade Federal de São Carlos" e "Ciência e Engenharia de Materiais";

2) Seleção do curso: na filtragem deste campo foram considerados todos os registros que continham em sua descrição os termos "materiais" e "doutorado". O termo "doutorado" foi considerado, pois apenas dois 


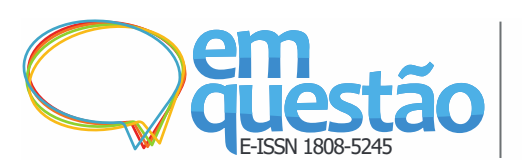

registros indicavam essa descrição para o campo "curso". Desta forma, foi considerado viável conferir o dado diretamente na Plataforma Lattes, atestando que os dois registros pertencem a acadêmicos que se formaram no PPGCEM/UFSCar;

3) Orientador: na filtragem deste campo foram desconsiderados todos os nomes que não pertencem ao PPGCEM/UFSCar, de acordo com a lista de docentes adquirida junto à Secretaria Geral de Planejamento e Desenvolvimento Institucionais (SPDI) da instituição.

Após a classificação restaram 993 registros. No entanto, para gerar a genealogia acadêmica pela segunda vez, foram desconsiderados os dados duplicados, ou seja, descendentes que possuem dupla titulação no programa. Para os descendentes que fizeram mestrado e doutorado no PPGCEM/UFSCar, adotou-se o critério baseado no processo da bibliometria das publicações científicas desses acadêmicos, considerando a titulação de mestrado.

Também foram desconsiderados os descendentes que não continham ID Lattes, um total de 367 registros, pois este é o parâmetro de entrada para se obter a genealogia acadêmica.Portanto, nesta etapa foram mapeados 586 descendentes diretos, com ID Lattes, do PPGCEM/UFSCar. Como pode ser constatado na Plataforma Lattes ao cadastrar uma Formação Acadêmica/Titulação, o sistema da plataforma faz um vínculo automático dos dados informados pelos usuários (CNPq, 2020).

No entanto, currículos mais antigos e desatualizados permanecem sem seus vínculos de relacionamento, exibindo apenas o nome do registro de orientação, o que, de acordo com Rossi, Damaceno e Mena-Chalco (2018),pode implicar na identificação dos relacionamentos.

A etapa quatro consiste em gerar a genealogia acadêmica pela segunda vez, a partir dos 586 descendentes diretos resultantes da etapa anterior. Os dados obtidos nessa etapa referem-se a todos os acadêmicos que pertencem à linhagem do PPGCEM/UFSCar de forma indireta,denominados de descendentes indiretos.

Posteriormente, na etapa 5, a partir dos resultados obtidos na etapa anterior, foi realizada a bibliometria das publicações científicas. Utilizou-se a ferramenta SyncLattes,desenvolvida por Matias (2015),que possibilitou obter os 
dados da produção científica de todos os descendentes. A ferramenta SyncLattes realiza a extração e o tratamento de metadados referentes as produções científicas indexadas nos Currículos Lattes. Durante o processo de extração, há a eliminação ou a combinação de informações duplicadas, evitando assim, erros na somatória das publicações científicas.

Esta ferramenta é muito utilizada na geração de indicadores de produção científica, que podem ser conferidos nos trabalhos de Maciel(2018) e Franco (2018). Como parâmetro para sua execução, precisa ser informado o IDLattes do pesquisador, portanto foram ignorados os registros sem ID Lattes válido.

Os dados resultantes da genealogia acadêmica também são disponibilizados em arquivos no formato de linguagem de marcação - XML e os resultados do SyncLattes em formato de texto.A partir deles, foi feita uma mineração de dados no VantagePoint, utilizando uma série de recursos que a ferramenta oferece, como filtros, listas e thesaurus em conjunto com o Microsoft Excel para construir indicadores.

\section{Resultados obtidos}

Após as execuções das etapas descritas na metodologia, os resultados obtidos baseados nos dados extraídos da Plataforma Lattes são apresentados e estruturados na seguinte ordem:

1. Análise da atuação do PPGCEM/UFSCar,

2. Análise dos dados dos descendentes do programa

3. Análise das produções científicas dos descendentes do programa.

Cabe observar que para o item 1 foram considerados todos os descendentes do programa, mesmo os sem ID Lattes válidos, pois o método de geração de grafos da herança acadêmica, mapeia a relação orientador-orientado. Para os itens 2 e 3, foram considerados somente os descendentes que tiveram os ID Lattes identificados pelo método de genealogia acadêmica, devido às ferramentas utilizadas. 


\subsection{Análise da atuação do PPGCEM/UFSCar}

O primeiro indicador extraído a partir da análise dos dados da genealogia acadêmica dos descendentes diretos do programa, foi o de contribuição do PPGCEM/UFSCar na formação de mestres e doutores.Pode ser observado na Figura 2 que, entre 1983 a 2019, foram formados 613 mestres. Já no período entre 1992 a 2019, foram formados 380 doutores, dentre os quais, 45 deles concluíram o mestrado e o doutorado no PPGCEM/UFSCar.

Figura 2 - Indicador de formação de recursos humanos pelo PPGCEM/UFSCar

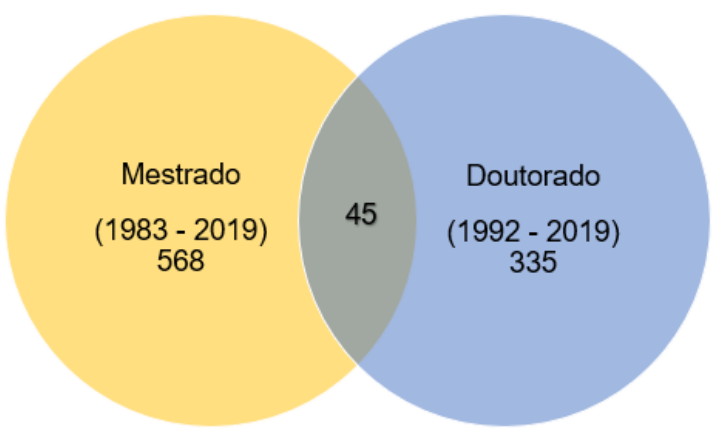

Fonte: Elaborado pelos autores

A partir desta informação buscou-se conhecer a atuação do programa na formação de recursos humanos ao longo do tempo e a partir disso, foi construído um indicador que representasse a distribuição desta formação durante o período estudado. A Figura 3 representa este indicador, e nela pode ser observado que nos anos de 2005, 2015 e 2016 o programa atingiu os melhores resultados, comparados com a série histórica.

Observa-se na Figura 3 que a partir de 1999 o programa manteve uma média aproximada de 38 formados por ano, ficando abaixo desta média entre os anos de 2007 até 2011, 2013 e 2014. Em 2002, 2003 e 2004, o número de doutores formados no programa, superou o de mestres, porém, em 2017, a quantidade de doutores formados teve queda, voltando a crescer em 2018 e 2019.

As variações dos resultados podem ter sido motivadas por vários fatores. Porém, como o objetivo deste indicador foi analisar a atuação do programa na formação de recursos humanos ao longo do tempo, este estudo não se aprofundou nos fatores que causaram essa variação. 

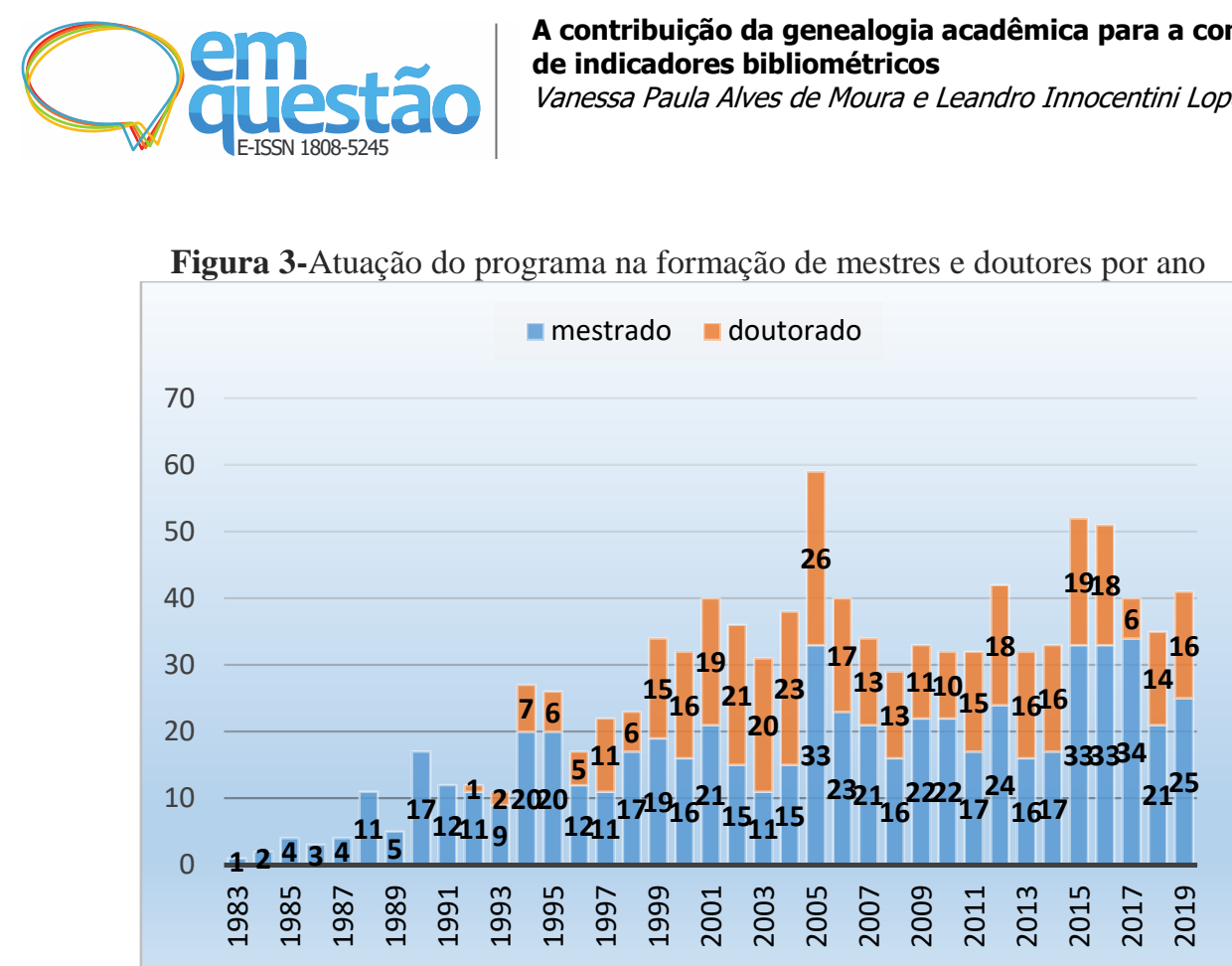

Fonte: Elaborado pelos autores

\subsection{Análise dos Descendentes}

Por meio do endereço profissional contido no Currículo Lattes foi possível identificar em quais tipos de instituições estão atuando os descendentes do PPGCEM/UFSCar. Para a construção deste indicador foram considerados todos os descendentes diretos e indiretos, totalizando 1503 registros. Deste total, 526 currículos não continham o endereço profissional indicado, representando aproximadamente $35 \%$. A análise foi realizada com base em 977 currículos,que representou aproximadamente $65 \%$ do total e, portanto, a maioria da amostra.

Como pode se observar na Figura 4 a maioria, um total de 623 descendentes, $63,77 \%$, atuam em universidades e instituições de ensino públicas como as universidades federais, estaduais e centros tecnológicos, a exemplo da Fatec (Faculdade de Tecnologia). Também é possível constatar que145 deles $(14,84 \%)$ estão atuando em empresas, destaque para ArcelorMittal Tubarão, Brasken S.A e Petrobrás, onde atuam mais de três descendentes. Já em universidades e instituições privadas, há uma concentração de 7,98\%, nos institutos federais, 4,61\%. Nas empresas nacionais de pesquisas, como a Embrapa, se concentram 2,15\% dos descendentes. Com pequena representatividade, há descendentes que indicaram em seus currículos endereços profissionais como hospital e polícia militar. 
Figura 4 - Atuação profissional dos descendentes

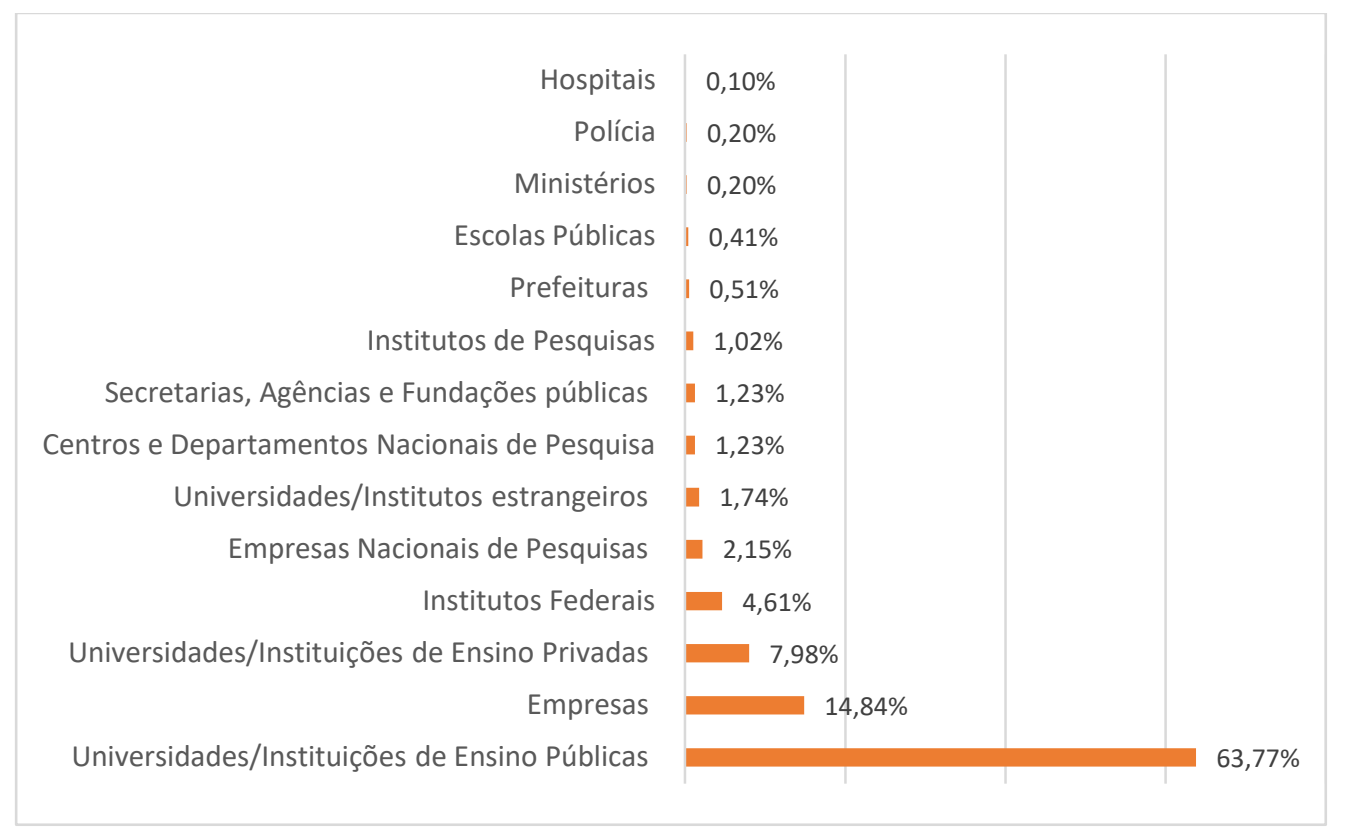

Fonte: Elaborado pelos autores

Por meio do endereço profissional, também foi possível identificar onde os descendentes do PPGCEM/UFSCar estão atuando no Brasil. Na Figura 5pode ser observado que estão presentes em praticamente todo território brasileiro, ou seja, 23 Estados. A maior concentração está em São Paulo, seguido por Paraná e Minas Gerais.

Esse indicador pode apontar o impacto do PPGCEM/UFSCar a nível nacional, além de poder contribuir para outras pesquisas que têm como objeto de estudo o desenvolvimento regional e a diminuição das diferenças científicotecnológico entre as regiões brasileiras e o quanto a área Engenharia de Materiais contribui para isso.

Observa-se, também na Figura 5, que a região nordeste possui 170 descendentes, 17,82\% do total, apesar da distância da sede do departamento do programa. Nesta região, destaca-se a Paraíba, com 51 descendentes, 30\%, do total da região. Em João Pessoa, encontra-se a Universidade Federal de Paraíba e em Campina Grande a Universidade Federal de Campina Grande, renomadas instituições na área de Engenharia de Materiais, o que pode explicar essa maior propagação do conhecimento. 
Figura 5 - Localização dos descendentes por regiões brasileiras

2

18

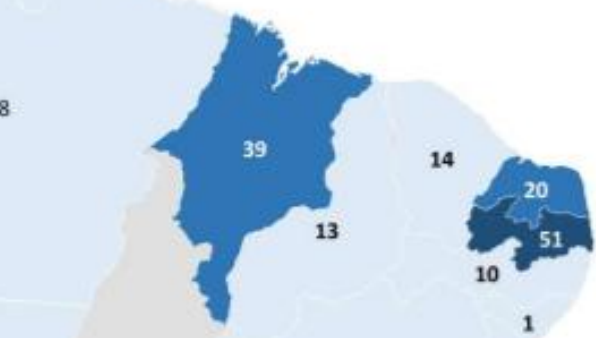

10

s

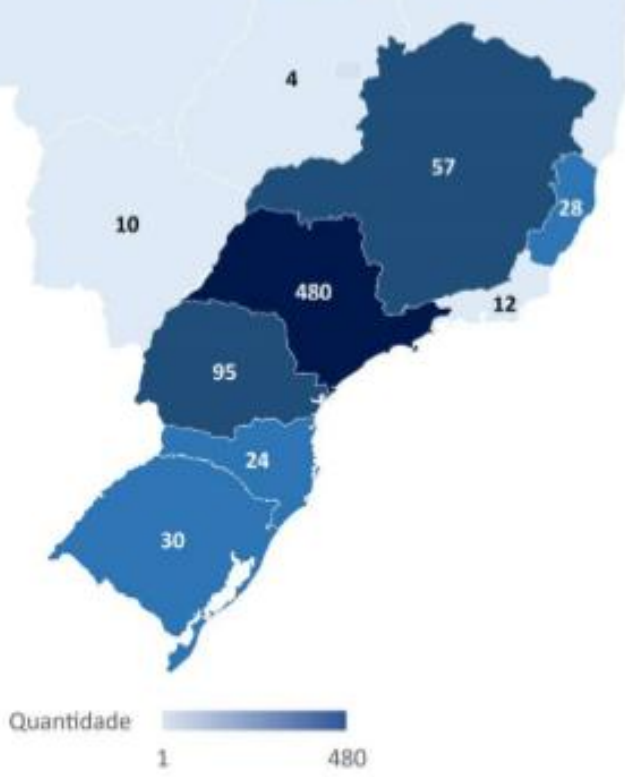

Fonte: Elaborado pelos autores

Como foi constatado pelo indicador das regiões brasileiras, a maioria dos descendentes do programa estão localizados no Estado de São Paulo, logo, entendeu-se a necessidade da criação de um indicador que detalhasse, a análise por cidade, para descobrir qual a cidade com maior concentração de descendentes do programa.

Na Figura 6, observa-se as cidades brasileiras que contêm o maior número de descendentes do PPGCEM/UFSCar. As cidades com menos de 10 descendentes tiveram seus números agrupados em "Outras cidades". 
Figura 6 - Cidades com maior concentração de descendentes

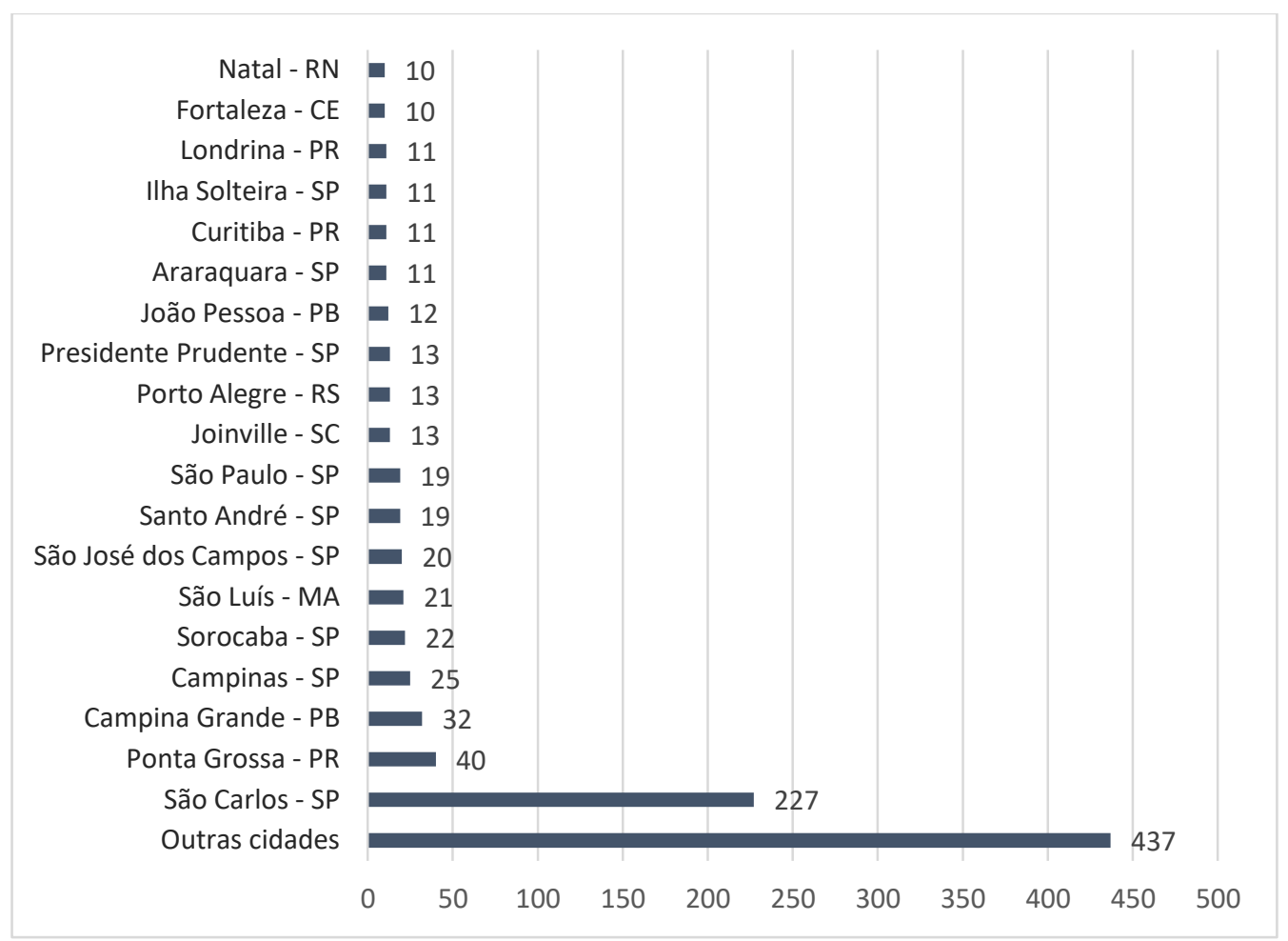

Fonte: Elaborado pelos autores.

Como ilustrado na Figura 6, a grande maioria encontra-se na cidade de São Carlos - SP, local sede do PPGCEM/UFSCar, onde se encontram 227 descendentes. As justificativas,hipotéticas, deste número são: a UFSCar tem sua sede em São Carlos; a cidade é considerada um polo tecnológico, o que propicia a absorção destes descendentes pelo mercado e, por fim, São Carlos também tem outras universidades.

Constata-se, uma concentração de descendentes na cidade de Ponta Grossa (PR) com 40 e Campina Grande (PB), com 32, Fortaleza (CE) e Natal (RN) com dez cada.

Diante deste cenário e baseado nos dados extraídos, buscou-se conhecer as principais instituições de ensino onde atuam os descendentes do PPGCEM/UFSCar. Pode-se observar na Figura 7que foram selecionadas as 10 instituições com maior número de descendentes. A UFSCar, instituição sede do programa, é onde se concentra o maior número de descendentes, com 167 . Na sequência, está a Universidade Estadual Paulista Júlio de Mesquita Filho (UNESP) com 41 descendentes e a Universidade de São Paulo (USP) com 38. 
Figura 7 - Atuação por Instituição

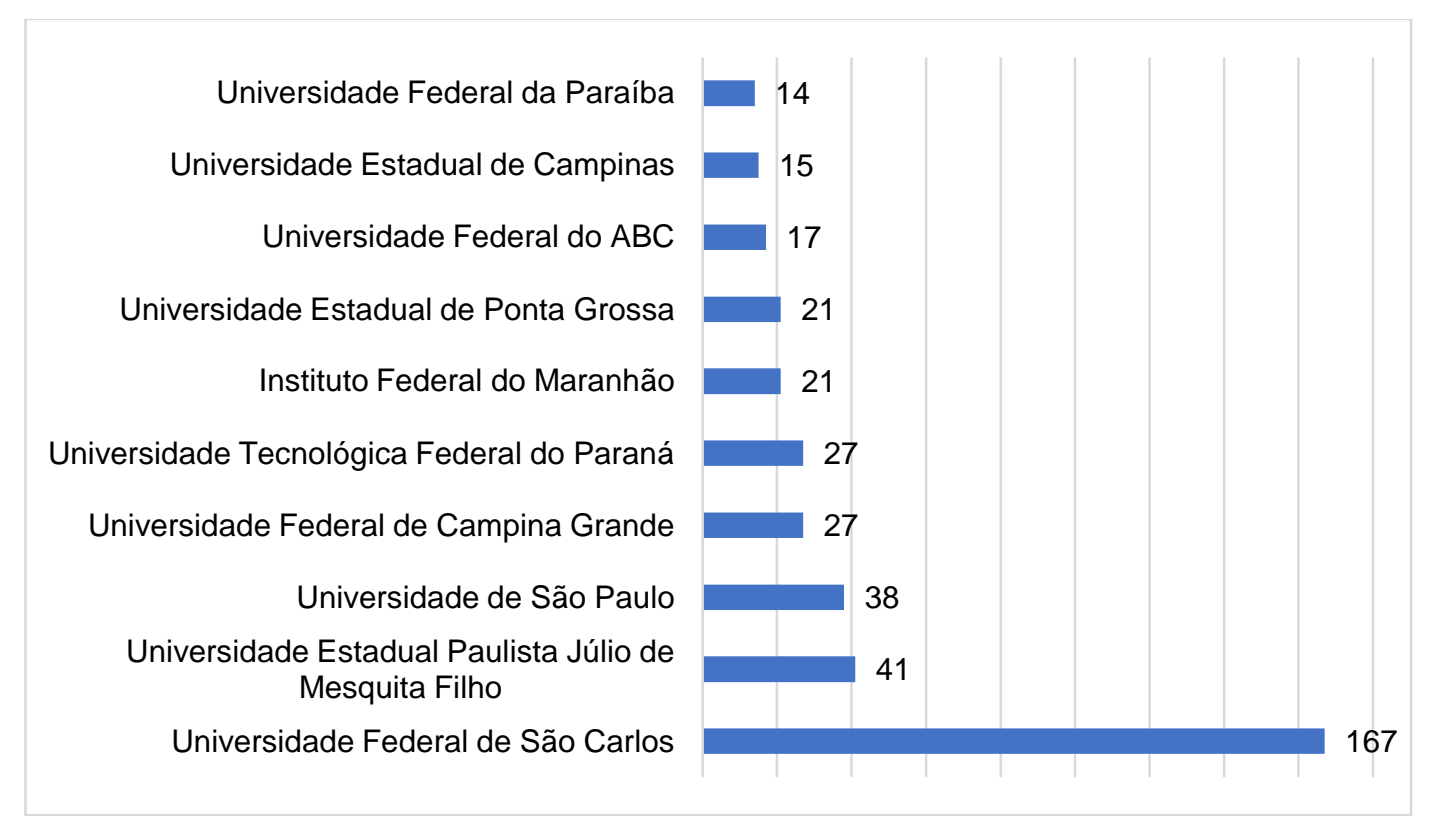

Fonte: Elaborado pelos autores

Destacam-se, também, a Universidade Federal de Campina Grande e a Universidade Federal da Paraíba. Juntas, somam 41 descendentes, representando $80 \%$ do total de descentes do programa que se encontram na Paraíba.

Além da instituição, foi possível detectar os departamentos onde esses descendentes diretos e indiretos atuam, indicador representado pela Figura 8. A classificação foi feita de acordo com os departamentos mais indicados no campo Órgão/Unidade contido no cadastro de endereço profissional do Currículo Lattes,porém, como este campo não é obrigatório, apenas 256 dos 997 descendentes tinham este campo preenchido, ou seja, 25,67\%. Os departamentos com duas ou menos indicações foram classificados em "outros". Embora a amostra não seja tão representativa, observa-se que 131 deles $(51,17 \%)$, consideram-se que atuam em um departamento de Engenharia de Materiais e Metalúrgica. 
Figura 8 - Atuação por departamento

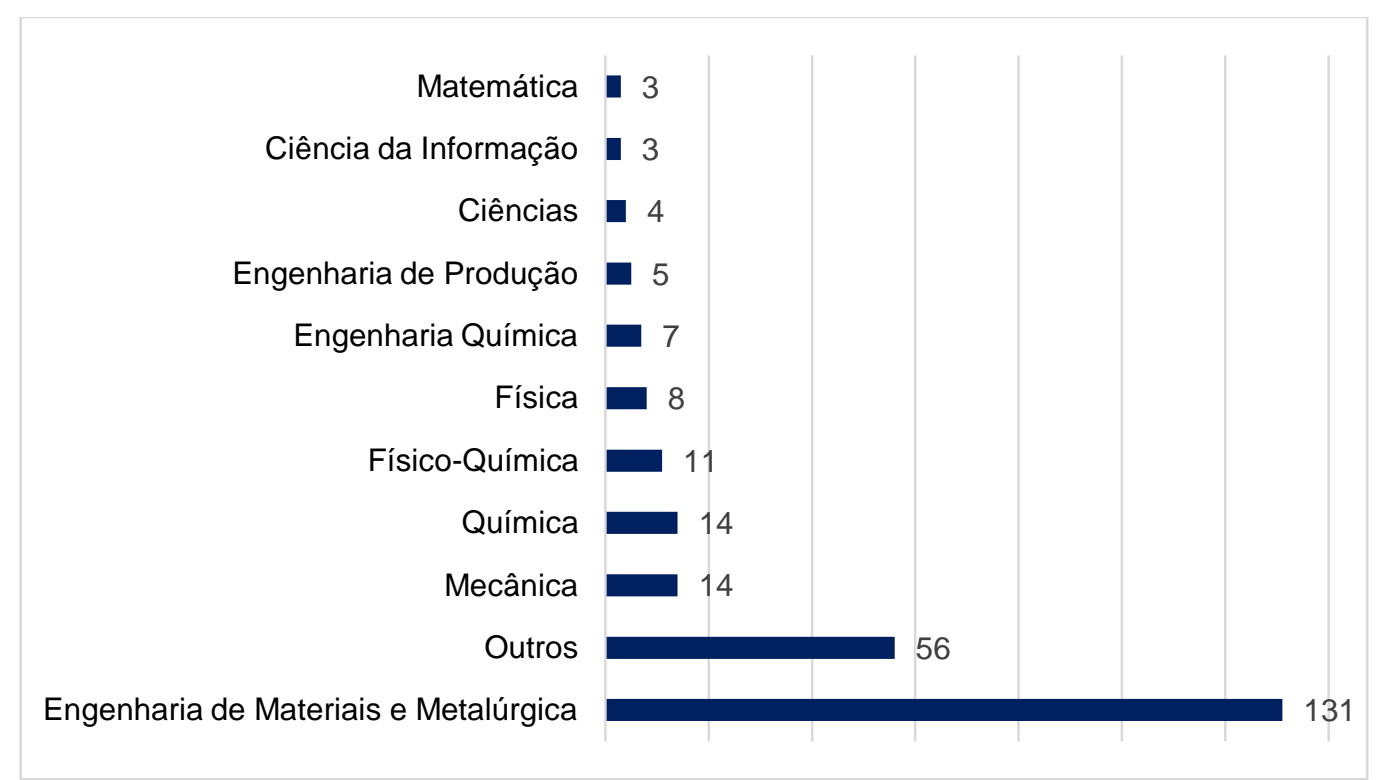

Fonte: Elaborado pelos autores

Como visto, a continuidade dos trabalhos de pesquisas desenvolvidos por um PPG depende do seguimento das pesquisas por seus formados. Desta forma, entende-se que a formação de mestres e doutores na área precisa ser contínua. Com base nisto, foi criado o indicador de área de formação dos descendentes indiretos,construído com o objetivo de avaliar se a continuação de formação na área está sendo mantida.

A partir do curso indicado pelos descendentes indiretos na Formação Acadêmica/Titulação em seus Currículos Lattes, foi feita uma classificação para mensurar as áreas mais indicadas por eles, as quais podem ser conferidas na Figura 9, onde 547 relacionaram a sua formação de mestrado ou doutorado com o termo "Engenharia de Materiais", ou seja, 45\%. Há aqueles que relacionaram suas áreas com o termo "materiais" totalizando 139 acadêmicos.

Este indicador possibilita observar a contribuição do PPGCEM/UFSCar na formação de recursos humanos na área de Engenharia de Materiais, o que pode contribuir na continuidade e no direcionamento das estratégias do programa. 
Figura 9 -Principais áreas de formação de mestrado

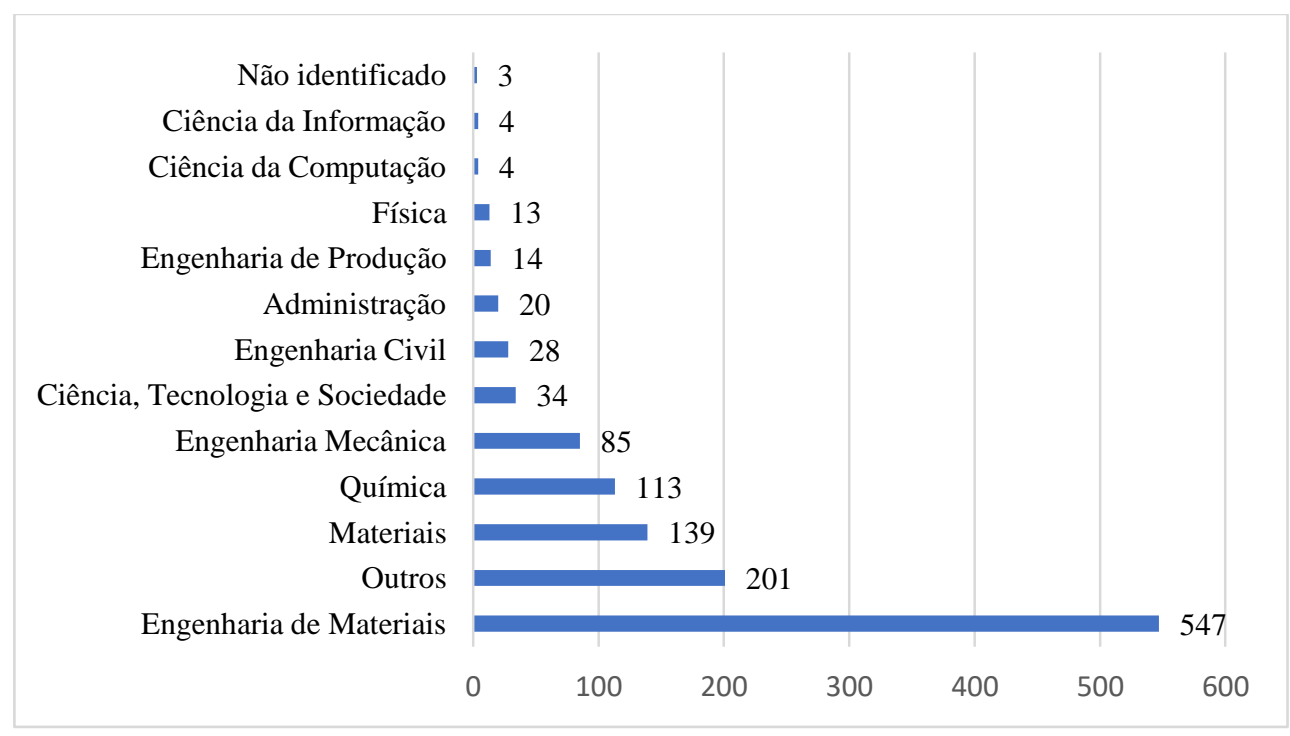

Fonte: Elaborado pelos autores.

\subsection{Análise da Produção Científica}

Para a construção dos indicadores de produção científica foram consideradas as publicações em periódicos e eventos científicos de todos descendentes e sementes do programa. Eles foram construídos a partir dos dados das publicações indexadas nos Currículos Lattes.

Do total de 1507 áreas apuradas, foi feita uma classificação que considerou a área de Engenharia de Materiais e Metalúrgica, as suas subáreas e especialidades, de acordo com áreas de conhecimento da Plataforma Sucupira (2020), separando-se a área do programa das demais áreas.

O primeiro indicador permite analisar os registros bibliográficos relativos aos artigos publicados em periódicos e eventos científicos, fazendo comparativo com as publicações realizadas na área de Engenharia de Materiais e Metalúrgica e nas demais áreas, entre os anos de 1979 até a data de extração de dados.

Após a extração dos dados foram apuradas 24.494 publicações em eventos científicos.Observando a Figura 10,a área de Engenharia de Materiais, que é a área de concentração do PPGCEM/UFSCar teve 8.486 publicações (34,6\% do total). Já nas outras áreas em conjunto, apurou-se 16.008 publicações, 
$65,34 \%$. Quanto às publicações de artigos em periódicos, estes somam um total de 12.789 e a área do programa representa 38,26\%. As demais áreas, 61,74\%.

Considerando o expressivo número de áreas indicadas, o número de publicações na área do referido programa se torna expressivo.

Figura 10 - Publicações e Eventos Científicos

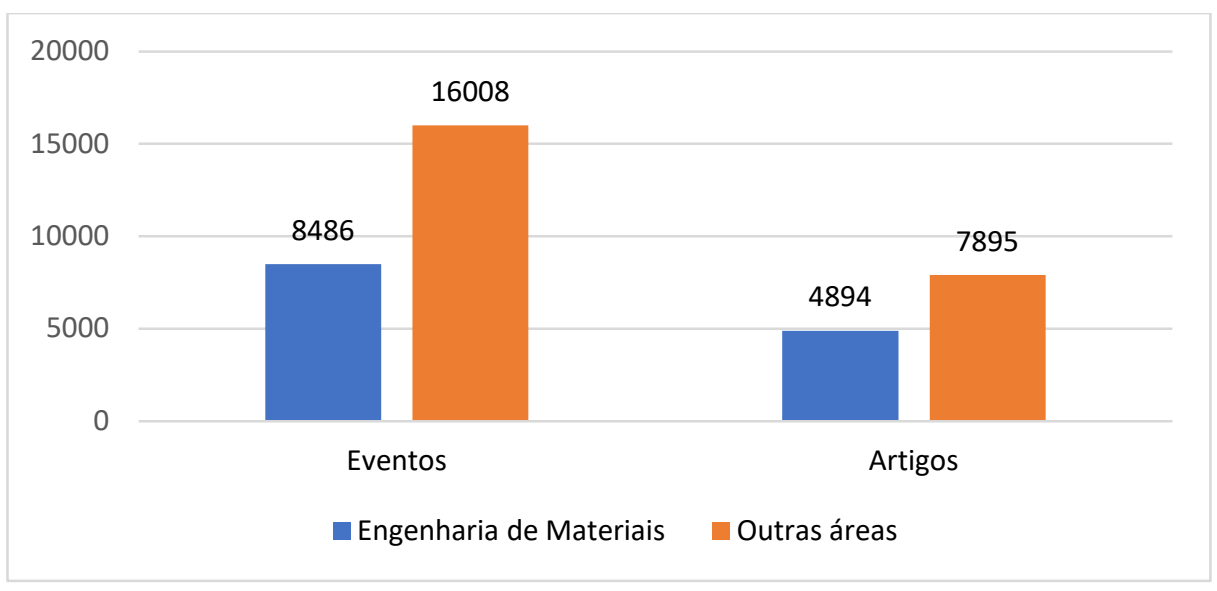

Fonte: Elaborado pelos autores

Para analisar a qualidade das produções intelectuais mais recentes dos descendentes do programa,foram utilizadas como amostras as publicações do período 2017 - 2020.Por meio da ferramenta VantagePoint,essas produções foram agrupadas de acordo com os veículos onde foram divulgadas. A partir do ISSN desses veículos foi feita uma pesquisa na Plataforma Sucupira para verificar suas classificações de acordo com os instrumentos avaliativos da CAPES.

Segundo a Plataforma Sucupira (2020), Qualis afere a qualidade dos artigos e de outros tipos de produção, a partir da análise de qualidade dos veículos de divulgação. No entanto,o novo modelo do Qualis Referência, encontra-se em fase de discussão e aprimoramento, desta forma, tomou-se como referência a classificação dos veículos no Quadriênio de 2013-2016, onde foram estabelecidos oito estratos de qualidade A1, A2, B1, B2, B3, B4, B5 e C, sendo A1 atribuído como nível mais elevado e C como nível mais baixo.

As publicações realizadas em veículos classificados como $\mathrm{C}$, ou que não tinham classificação, foram consideradas como "Demais Publicações". 
$\mathrm{Na}$ área de Engenharia de Materiais e Metalúrgica somaram-se 547 publicações,as quais foram classificados de acordo com a Figura 11, onde se pode constatar que 191 publicações foram divulgadas em veículos classificados com Qualis A1 e 85 com Qualis A2, representando 50\% do total das publicações de todo o período em veículos de referência.

Figura 11 -Publicações por Qualis Periódico

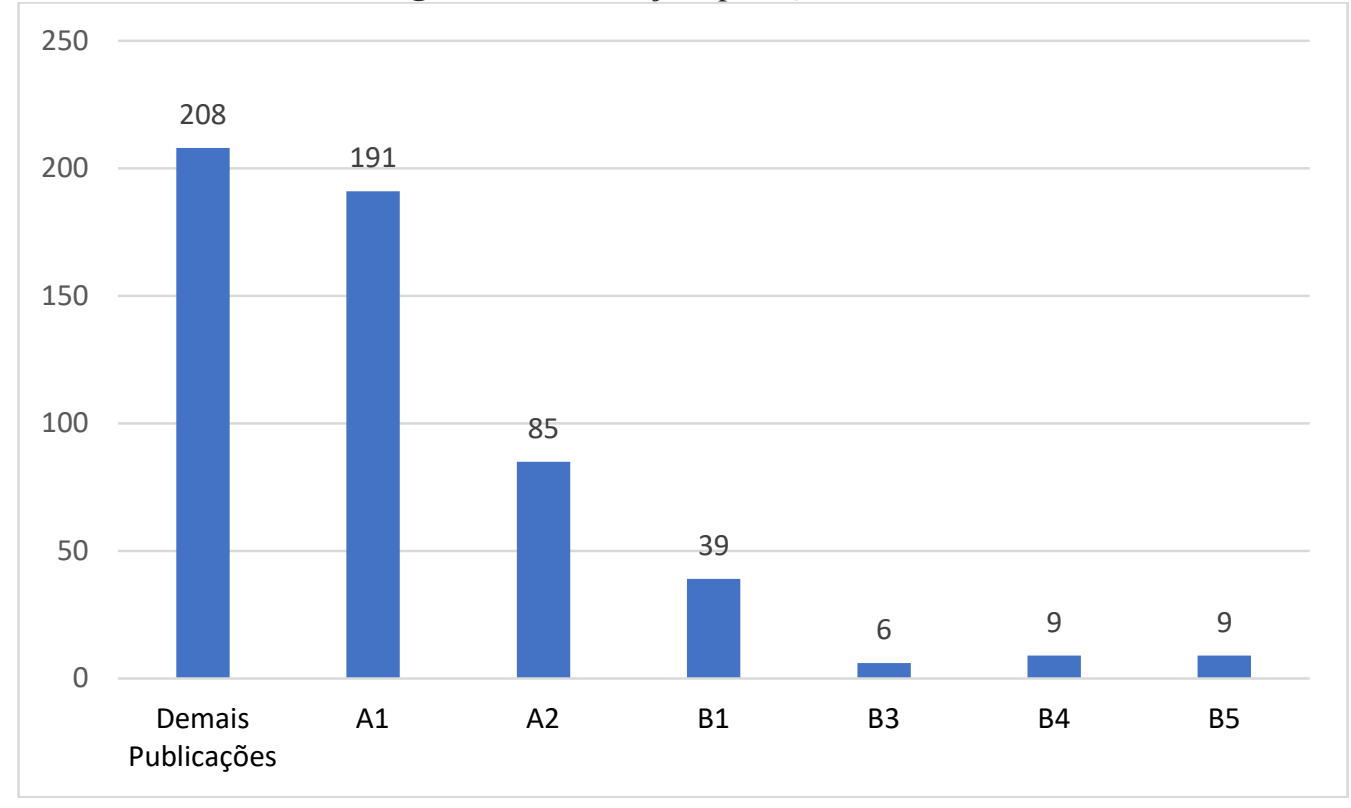

Fonte: Elaborado pelos autores

\section{Considerações finais}

Os resultados da genealogia acadêmica por meio do método de identificação de grafos de herança acadêmica, não foi desenvolvido com o propósito de analisar as produções científicas. Contudo, a partir deste mapeamento foi possível identificar os dados necessários para a realização desta análise.

A união da genealogia acadêmica com a bibliometria da produção científica permitiu construir um cenário amplo e repleto de informações do PPGCEM/UFSCar e sobre seus descendentes, que podem contribuir para a análise da atuação do departamento e de seus docentes, de acordo com a necessidade do programa, além de propiciar ao programa informações que podem não ser do seu conhecimento, como,por exemplo, sobre o fluxo do conhecimento gerado no programa entre seus descendentes, onde se encontram e suas contribuições para a área. 
Por meio das análises, foram gerados vários indicadores, e 11 deles foram escolhidos na divulgação dos resultados dessa pesquisa.

Considera-se, que o objetivo desta pesquisa foi atingido. Em trabalhos futuros,podem ser realizadas análises de outros PPG's, análises bibliométricas para a construção de redes de coautoria e, por fim, estender os horizontes para a criação de indicadores que contribuam para a melhoria constante dos programas.

\section{Referências}

ANDRAOS, J. Scientific genealogies of physical and mechanistic organic chemists. CanadianJournal of Chemistry, [S.l.], v. 83, n. 9, p. 1400-1414, 2005.

ARAÚJO, C. A. À. A ciência como forma de conhecimento. Ciências \& Cognição, Belo Horizonte, v. 8, n.11, p. 127-142, 2006.

ROSA, F. ; BARROS, S. Comunicação científica: reflexões preliminares para o GT "relevância dos livros acadêmicos na comunicação da pesquisa. [S.l.]: Scielo, 2018. Disponível em:<https://www.scielo20.org/redescielo/wpcontent/uploads/sites/2/2018/07/ROSA-F_-BARROSS.-ComunicacaoCientifica.pdf>. Acesso em: 2 abr. 2019.

BIBLIOTECA DA FEUP. Publicação Científica: Contextos e evolução.Guias Temáticos de Apoio. Porto: Faculdade de Engenharia Universidade do Porto, 2019. Disponível em: <https://feup.libguides.com/publicacaocientifica/contextos>. Acesso em: 17 set. 2019.

CHIBENI, S. S. O que é ciência? Campinas: Departamento de Filosofia da Unicamp, 2019. Disponível em:

<https://www.unicamp.br/ chibeni/textosdidaticos/ciencia.pdf>. Acesso em: 17 set. 2019.

CNPq. O que é a Plataforma Lattes: Sobre a Plataforma. Brasília: CNPq, 2019. Disponível em:<http://memoria.cnpq.br/web/portal-lattes/sobre-aplataforma>. Acesso em: 2 abr. 2019.

CNPq. Currículo Lattes: Cadastro de Formação Acadêmica/Titulação. Brasília: CNPq, 2019. Disponível em: <http://lattes.cnpq.br/>. Acesso em: 28 mai. 2020.

DAMACENO, R.J.P.; ROSSI, L.; MENA-CHALCO, J.P. Identificação do grafo de genealogia acadêmica de pesquisadores: Uma abordagem baseada na Plataforma Lattes. In: BRAZILIAN SYMPOSIUM ON DATABASES, 32. 2017, Uberlândia.Anais[...]. Uberlândia: SBBD, 2017. p. 76-87. 
DAMACENO, R. J. P. et.al. The Brazilian academic genealogy: evidence of advisor-advisee relationships through quantitative analysis. Scientometrics, [S.l.]: v. 119, n. 01, p. 303-333, 2019.

DORES, W. et.al.Building the Brazilian Academic Genealogy Tree.Belo Horizonte: Universidade Federal de Minas Gerais, 2017. Disponível em:<https:// https://homepages.dcc.ufmg.br/ fabricio/download/short-tpdlgenealogy_sub.pdf $>$. Acesso em 01 dez. 2019.

FARIA, L. I. L. de. Prospecção tecnológica em materiais: aumento da eficiência do tratamento bibliométrico. Aplicação na análise de tratamentos de superfície resistentes ao desgaste. 2001. Tese (Doutorado em Ciência e Engenharia de Materiais) - Universidade Federal de São Carlos, São Carlos, 2001.

FRANCO, N. M G. Análise relacional intrainstitucional: redes de coautoria e acoplamento de autores, 2018. 134 f. Dissertação (Mestrado) - Programa de Pós-Graduação em Ciência da Informação, Universidade Federal de São Carlos, São Carlos, 2018.

INSTITUTO DE CIÊNCIAS BIOLÓGICAS. Introdução à Análise de Genealogias.Belo Horizonte: Instituto de Ciências Biológicas da Universidade Federal de Minas Gerais, 2019. Disponível em:

<https://www2.icb.ufmg.br/grad/genetica/heredogramas.htm>. Acesso em 01 dez. 2019.

LE COADIC, Y.-F. A ciência da informação. Brasília,Briquet de Lemos, 1996.

LIMA, W. T. A. Avaliação de egressos da pós-graduação estrito senso: Política de monitoramento de egressos internacionais. [S.l.]: Scielo, 2015.Disponível em: <https://www.scielo.br/scielo.php?pid=S010069912015000800026\&script=sci_arttext\&tlng=pt >. Acesso em $01 \mathrm{dez} .2019$.

MACIEL, R. S. A Plataforma Lattes como recurso estratégico para a gestãodos Programas de Pós-Graduação: uma análise baseada na produção de artigos científicos, 2018. 183 p.Dissertação (Mestrado) - Programa de PósGraduação emCiência da Informação, Universidade Federal de São Carlos, São Carlos, 2018.

MATIAS, M. S. O.Base referencial para o povoamento de repositórios institucionais: coleta automatizada de metadados da Plataforma Lattes. 2015. 94 f. Dissertação (Mestrado) - Programa de Pós-Graduação em Gestão de Organizações e Sistemas Públicos, Universidade Federal de São Carlos, São Carlos, 2015.

MOREIRA, T. H. et. al. Árvores genealógicas acadêmicas como estratégia para análises de processo de orientação. Pesquisa Brasileira em Ciência da

Informação eBiblioteconomia, João Pessoa, v. 13, n. 2, p. 246-254, 2018. 
OLIVEIRA, C. A. et. al. Genealogia acadêmica dos pesquisadores da área de Ciência da Informação: um estudo sobre os bolsistas de produtividade em pesquisa (PQ-CNPq). Em Questão, Porto Alegre, v.24, n. edição especial 6. p. 278-298, 2018.

PLATAFORMA SUCUPIRA. Áreas de Avaliação/Conhecimento. Disponível em:https://sucupira.capes.gov.br/sucupira/public/consultas/coleta/programa/qua ntitativos/quantitativoles.jsf?areaAvaliacao $=12$ \&areaConhecimento $=30300002$. Acesso em: 01 fev. 2020.

PLATAFORMA SUCUPIRA. Qualis Periódicos. Disponível em: https://sucupira.capes.gov.br/sucupira/public/index.jsf.Acesso em: 01 fev. 2020.

RIBEIRO, R. J. Os critérios da avaliação. Brasília, CAPES, Brasília, 2007. Disponível em:

https://www1.capes.gov.br/images/stories/download/artigos/Artigo_10_08_07.p df . Acesso em: 24 abr. 2019.

ROSSI, L.; MENA-CHALCO, J. P. Caracterização de árvores de genealogia acadêmica por meio de métricas em grafos. In: BRAZILIAN WORKSHOP ON SOCIAL NETWORK ANALYSIS AND MINING (BRASNAM), 3. , 2014, Brasília. Anais [...]. Porto Alegre: Sociedade Brasileira de Computação, 2014. p.21-32.

ROSSI, L.; DAMACENO, R.; MENA-CHALCO, J. Genealogia acadêmica: Um novo olhar sobre impacto acadêmico de pesquisadores. Revista Parcerias Estratégicas, Brasília, v.23. n.47, p. 197-212, 2018.

SAVIGNANO, V. Um aniversário da Engenharia de Materiais no Brasil: 40 anos do DEMa da UFSCar. BrazilianMaterialsReserch Society, [S.l.], 2012. Disponível em: <https://www.sbpmat.org.br/pt/um-aniversario-da-engenhariade-materiais-no-brasil-40-anos-do-dema-da-ufscar/>. Acesso em: 24 set. 2019.

SILVA, J. L. C.; FREIRE, G. H. A. Um olhar sobre a origem da ciência da informação: indícios embrionários para sua caracterização identitária. Revista Eletrônica de Biblioteconomia e Ciência da Informação,[S.l.], v. 17, n. 33, 2012.

SPINAK, E.; PACKER, A. L. 350 anos de publicação científica: desde o "Journal des Sçavans" e "PhilosophicalTransactions" até o SciELO, [S.l.]: Scielo em Perspectiva, 2015. Disponível em: https://blog.scielo.org/blog/2015/03/05/350-anos-de-publicacao-cientificadesde-o-journal-des-scavans-e-philosophical-transactions-ate-oscielo/\#.XYE2ei5Kjcs. Acesso em: 13 set. 2019.

SOUZA, L. G. De. Relação entre ciência \& pesquisa, enContribuciones a lasCienciasSociales, Texcoco: Eumed, 2011. 
SUGIMOTO, C. R. et al. Academic Genealogy as an Indicator of Interdisciplinarity: An Examination of Dissertation Networks in Library and Information Science, Journal of the American Society for Information Science, [S.L], v.62, p. 1808-1828, 2011.

SUGIMOTO, C. R. Beyond bibliometrics. In:Political Geography. London: Massachusetts Institute of Technology, 2014.

UNESCO, C. N. Da. Ciência para a sociedade.Comissão Nacional da Unesco. Disponível em: https://www.unescoportugal.mne.pt/pt/temas/ciencia-para-umfuturo-sustentavel/ciencia-para-a-sociedade. Acesso em: 19 fev. 2020.

VELHO, L. Cuidado com os rankings científicos.Porque deve-se temer as avaliações sobre quem produz mais ciência, e como essas avaliações discriminam o Terceiro Mundo.Agência de Notícias Prometeu, Campinas, 2011. Disponível em: http://www.prometeu.com.br/bb-lea.htm. Acesso em: 13 set. 2019.

\title{
The contribution of academic genealogy to the building of bibliometric indicators
}

\begin{abstract}
The measurement of scientific production through bibliometrics, which allows assessing the performance of researchers and the impacts of their production, is an object of study widely explored in Information Science. Academic genealogy, on the other hand, allows us to study the intellectual heritage resulting from the relationship between supervisors and their students. Recent studies in Brazil on academic genealogy use the Lattes Platform as a database, extracting information that can be used for various analyzes. Therefore, this work aims to investigate whether the application of academic genealogy and bibliometric analysis together can bring complementary information about the performance of a Postgraduate Program in the training of researchers and scientific production, carrying out a case study about the Program Graduate Program in Materials Engineering at the University of São Paulo. Federal University of São Carlos. The methodology used is based on the method of identifying academic genealogy charts in conjunction with the SyncLattes, VantagePoint and Microsoft Excel tools. As results, bibliometric indicators about the program and its descendants are presented, which can contribute to the development of self-assessment strategies and performance of graduate programs.
\end{abstract}

Keywords:Bibliometrics. Academic genealogy. Scientific production. Science and technology indicators. Engineering II.

Recebido: 30/03/2020

Aceito: $23 / 06 / 2020$ 


\section{Como citar}

MOURA, Vanessa Paula Alves de.;FARIA, Leandro Innocentini Lopes de. A contribuição da genealogia acadêmica para a construção de indicadores

bibliométricos. Em Questão, Porto Alegre, v.27, n. 1, p. 336-360, 2021. Doi: https://doi.org/10.19132/1808-5245271.336-360 